\title{
Supersymmetry without hermiticity
}

\author{
Miloslav Znojil| \\ Ústav jaderné fyziky AV ČR, 25068 Řě̌, Czech Republic, \\ Francesco Cannataf \\ Dipartimento di Fisica dell'Universita' and INFN, via Irnerio 46, I40126 Bologna, \\ Italy, \\ Bijan Bagchi \\ Department of Applied Mathematics, University of Calcutta, 92 Acharya Prafulla \\ Chandra Road, Calcutta 700 009, India, \\ and \\ Rajkumar Roychoudhury't \\ Physics and Applied Mathematics Unit, Indian Statistical Institute, Calcutta \\ 700035, India
}

\footnotetext{
${ }^{1}$ e-mail: znojil@ujf.cas.cz

${ }^{2}$ e-mail: cannata@bo.infn.it

${ }^{3}$ e-mail: bbagchi@cucc.ernet.in

${ }^{4}$ e-mail: raj@www.isical.ac.in
} 


\section{Abstract}

A new model of supersymmetry between bosons and fermions is proposed. Its representation space is spanned by states with $\mathcal{P} \mathcal{T}$ symmetry and real energies but the inter-related partner Hamiltonians themselves remain complex and non-Hermitian. The formalism admits vanishing Witten index. 


\section{Introduction}

Supersymmetry (SUSY, [1]) offers a chance of unification of bosons with fermions in various branches of physics [2]. Mathematically, it mixes the commutators and anticommutators in a single (so called graded) Lie algebra. In its simplest form $\operatorname{sl}(1 / 1)$ this symmetry algebra is generated by two supercharges $\mathcal{Q}, \tilde{\mathcal{Q}}$ and a Hermitian Hamiltonian $\mathcal{H}$. These three generators are related by the anticommutation rules

$$
\{\mathcal{Q}, \tilde{\mathcal{Q}}\}=\mathcal{H}, \quad\{\mathcal{Q}, \mathcal{Q}\}=\{\tilde{\mathcal{Q}}, \tilde{\mathcal{Q}}\}=0
$$

and commutativity

$$
[\mathcal{H}, \mathcal{Q}]=[\mathcal{H}, \tilde{\mathcal{Q}}]=0
$$

SUSY finds an enormous appeal in particle physics and field theory [3]. In this application, unfortunately, there exists the strong conflict between the theory and experiment. Due to the continuing absence of observation of any bosonic-fermionic degenerate multiplets, any form of SUSY must be badly broken as a consequence 《4.

Attempts to resolve the latter physical problem encounter nontrivial mathematical difficulties [5]. Possible mechanisms of SUSY breaking are currently being exposed to an intensive research [6]. One of the most feasible ways of their analysis is offered by the representation of SUSY algebras in a zero-dimensional field theory, i.e., in quantum mechanics [7, 8]. We intend to contribute to this effort by weakening certain assumptions concerning, first of all, the hermiticity of $H$.

\section{PT symmetric non-Hermitian models}

Conventional supersymmetric quantum mechanics does not immediately admit complex potentials. Only recently, the first attempts in this direction have been made [9, 10]. A significant improvement of our understanding of the underlying complex dynamics has been offered by Bender and Boettcher [11]. Within their so called 
$\mathcal{P} \mathcal{T}$ symmetric quantum mechanics [12] they proposed a replacement of the usual hermiticity of the Hamiltonian $H$ by its mere commutativity with the product of the parity $\mathcal{P}$ and the time reversal $\mathcal{T}$

$$
H \mathcal{P} \mathcal{T}=\mathcal{P} \mathcal{T} H
$$

It has been shown by several methods that one may get real energy spectrum in many different systems of this type [13]. Similar analytic assumptions made in connection with quantized systems is not so unusual in the mathematically oriented literature [14]. Its appeal in physical applications is also undeniable and ranges from perturbative [15] and semiclassical methods and considerations [16] up to the practical computation of resonances [17. In such a setting, the construction of representations of SUSY algebras encounters several new challenges. Some of them will be addressed in what follows.

\subsection{Facilitated normalization}

For a concise exposition of some of the related open questions let us first recall a quartic partially solvable potential

$$
V_{1}(r)=-r^{4}+2 i r
$$

of ref. 18. Its one-dimensional Schrödinger equation

$$
-\frac{d^{2}}{d r^{2}} \Psi(r)+V_{1}(r) \Psi_{1}(r)=E_{1} \Psi_{1}(r), \quad r \in(-\infty, \infty)
$$

possesses a formal solution at zero energy $E_{1}=0$. Forgetting, for the time being, that this solution is not normalizable in the usual sense,

$$
\Psi_{1}^{(0)}(r)=\exp \left(\frac{i r^{3}}{3}\right) \notin L_{2}(-\infty, \infty)
$$

we can construct its superpotential

$$
W_{1}(r)=-\left[\frac{d}{d r} \Psi_{1}^{(0)}(r)\right] / \Psi_{1}^{(0)}(r)=-i r^{2}
$$


and derive formally the supersymmetric partner potential [7]

$$
V_{2}(r)=W_{1}^{2}(r)+W_{1}^{\prime}(r)=W_{2}^{2}(r)-W_{2}^{\prime}(r)=-r^{4}-2 i r, \quad r \in(-\infty, \infty)
$$

as well as the parallel ground-state-like solution

$$
\Psi_{2}^{(0)}(r)=\exp \left(\frac{-i r^{3}}{3}\right) \notin L_{2}(-\infty, \infty) .
$$

Obviously, in such a model the formal SUSY transformation $1 \rightarrow 2$ degenerates to the mere time reversal represented, for our present purposes, by the above-mentioned operator $\mathcal{T}$ which replaces $i$ by $-i$ [1],

$$
\mathcal{T} \Psi_{2}^{(0)}(r)=\Psi_{1}^{(0)}(r), \quad \mathcal{T} V_{2}(r) \mathcal{T}=V_{1}(r)
$$

Within the less naive framework of the $\mathcal{P} \mathcal{T}$ symmetric quantum mechanics the latter example proves better understood. Firstly, in the light of the analyticity of our model we can restore the normalizability of its wave functions (3) and (4) by suitable deformations of the coordinate axis in complex plane [12]. This can be achieved by the mere shifts

$$
r=r_{1,2}(x)=x \pm i \varepsilon, \quad \varepsilon>0, \quad x \in(-\infty, \infty)
$$

of the respective integration paths. This guarantees that the wave functions become asymptotically vanishing as required, $\Psi_{j}^{(0)}\left[r_{j}( \pm \infty)\right] \rightarrow 0$. Unfortunately, we have to pay a high price. After one verifies that

$$
V_{2}=W_{2}^{2}-W_{2}^{\prime}=-x^{4}+4 i \varepsilon x^{3}+\ldots \neq W_{1}^{2}+W_{1}^{\prime}=-x^{4}-4 i \varepsilon x^{3}+\ldots
$$

we have to conclude that our two new, $\mathcal{P} \mathcal{T}$ symmetrized interactions $V_{1,2}$ cease to be inter-related by a supersymmetry.

In what follows we intend to re-solve the puzzle. In essence, we shall generalize the original Witten's quantum mechanical construction [4]. Our attention will be paid to situations where the above-exemplified loss of a SUSY partnership could be 
re-established anew. In brief, we shall propose an entirely new representation of the supersymmetric algebra within the framework of the $\mathcal{P} \mathcal{T}$ symmetric quantum mechanics.

\subsection{A toy model without SUSY}

We intend to introduce our proposal via a few explicit examples. Particular attention will be paid to the two manifestly $\mathcal{P} \mathcal{T}$ symmetric potentials

$$
\begin{gathered}
V^{(-)}(x)=-4 i(x-i \varepsilon)-(x-i \varepsilon)^{4}, \\
V^{(+)}(x)=\frac{2}{(x+i \varepsilon)^{2}}-(x+i \varepsilon)^{4} .
\end{gathered}
$$

Their doublet resembles the previous pair by the similar choice of the respective domains $r_{( \pm)}(x)=x \pm i \varepsilon$. Conveniently, both their shifts are equal and given by the same positive constant $\varepsilon>0$. Our new examples (5) and (6) also exhibit the so called quasi-exact solvability revealed in refs. [18] and [19], respectively. Meaning just that a few exact bound states remain at our disposal in an elementary form [20], this property offers us the two exact zero-energy bound-state solutions

$$
\begin{gathered}
\psi^{(-)}(x)=(x-i \varepsilon) \exp \left(-i \frac{(x-i \varepsilon)^{3}}{3}\right) \in L_{2}(-\infty, \infty), \\
\psi^{(+)}(x)=\frac{1}{x+i \varepsilon} \exp \left(+i \frac{(x+i \varepsilon)^{3}}{3}\right) \in L_{2}(-\infty, \infty)
\end{gathered}
$$

representing, presumably, ground states. Both these wave functions are bounded and normalizable if and only if their common real parameter $\varepsilon$ is positive. This is similar to our previous illustration while, in contrast, the new superpotentials

$$
W^{( \pm)}(x)=-\left[\frac{d}{d x} \psi^{( \pm)}(x)\right] / \psi^{( \pm)}(x)= \pm\left[\frac{1}{x \pm i \varepsilon}-i(x \pm i \varepsilon)^{2}\right]
$$

differ more than just by an overall sign.

In the unphysical extreme of the vanishing parameter $\varepsilon \rightarrow 0$ we would arrive at

the standard SUSY connecting our two Hamiltonians $H^{( \pm)}$but no serious progress 
seems to have been achieved. We again "stumble" over the normalizability of our wave functions which would be lost in the SUSY limit. Still, there is a difference. We are going to show below that our new doublet of models can be supersymmetrized after one modifies the usual recipe.

\section{$3 \quad$ PT symmetric supersymmetry}

A key observation of our present proposal is that many $\mathcal{P} \mathcal{T}$ symmetric systems are defined off the real axis. Boundary conditions $\lim _{|r| \rightarrow \infty} \psi(r)=0$ are in general located within wedges bounded by Stokes' lines [18]. Locally, the paths of integration can be deformed whenever necessary. In contrast to our introductory examples $V_{1,2}$, the mere "time-reflection" conjugation $\mathcal{T}$ itself does not now map our new Hamiltonians $H^{( \pm)}$upon each other. Still, an active use of $\mathcal{T}$ will be a key ingredient in our forthcoming construction.

\subsection{Innovated factorization of Hamiltonians}

With our functions (7) taken just as a particular illustration, let us now assume their arbitrary form and introduce the four related operators

$$
A^{( \pm)}=\frac{d}{d x}+W^{( \pm)}(x), \quad B^{( \pm)}=-\frac{d}{d x}+W^{( \pm)}(x)
$$

which induce the traditional Riccati-equation formulae

$$
H^{( \pm)}=B^{( \pm)} A^{( \pm)}=-\partial_{x}^{2}+\left[W^{( \pm)}(x)\right]^{2}-\left[W^{( \pm)}(x)\right]^{\prime}
$$

We have to fit these two Hamiltonians into a generalized SUSY scheme of the type (1) + (2). In the first step, we explored reordered products. Returning to our explicit

examples $V^{( \pm)}$for inspiration, we did not succeed in the ${ }^{(+)}$-superscripted case at all. Fortunately, in the second case we were able to verify by immediate insertions 
the strict validity of the only slightly nonstandard rule

$$
H^{(+)}=\mathcal{T} A^{(-)} B^{(-)} \mathcal{T}
$$

This formula is, in a way, our central point. Indeed, once we arrange our doublet of Hamiltonians into the following two-dimensional array

$$
\mathcal{H}=\left[\begin{array}{cc}
H^{(-)} & 0 \\
0 & H^{(+)}
\end{array}\right]=\left[\begin{array}{cc}
B^{(-)} A^{(-)} & 0 \\
0 & \mathcal{T} A^{(-)} B^{(-)} \mathcal{T}
\end{array}\right]
$$

we recover immediately all the necessary SUSY rules (11) and (2), provided only that we introduce the following modified representation of the supercharges,

$$
\mathcal{Q}=\left[\begin{array}{cc}
0 & 0 \\
\mathcal{T} A^{(-)} & 0
\end{array}\right], \quad \tilde{\mathcal{Q}}=\left[\begin{array}{cc}
0 & B^{(-)} \mathcal{T} \\
0 & 0
\end{array}\right]
$$

In contrast to the usual SUSY constructions our new supercharges are not correlated by any Hermitian conjugation anymore. This is our main methodological gain. A new hope is created that some "no-go" theorems of the traditional Hermitian theories could be overcome within our new SUSY framework.

A key technical difficulty with this hope lies in its dependence on the specific choice of our example. Fortunately, one can re-analyze our fundamental rearrangement in the more general context where the explicit form

$$
\left[W^{(+)}\right]^{2}-\left[W^{(+)}\right]^{\prime}=\left\{\left[W^{(-)}\right]^{2}+\left[W^{(-)}\right]^{\prime}\right\}^{*}
$$

of eq. (8) may be called a ladder equation. It glues superpotentials in the language of the higher order SUSY [21]. An explicit solution of the latter equation exists and can be expressed in the parametric form using an arbitrary complex function $f(x)$,

$$
W^{(+)}=\frac{f^{\prime}}{2 f}-f, \quad W^{(-)}=-\frac{f^{\prime *}}{2 f^{*}}-f^{*}
$$

It is worth noticing that with the shape invariant $f(x)=-i \lambda$ sech $\mu x$, one rediscovers the amazing though very special relationship between the non-Hermitian 
and Hermitian exactly solvable models of ref. [22]. In the latter class of examples with the purely imaginary $f(x)$ our "new SUSY" coincides with the ordinary, "classical SUSY" since the lower Hamiltonian remains real, $H^{(+)}=\mathcal{T} H^{(+)} \mathcal{T}$. With

arbitrary $f(x)$ equation (10) suggests that a certain modified Darboux transformation [23] is at work for $H^{(+)} \neq \mathcal{T} H^{(+)} \mathcal{T}$. In this way, also another, "fully complex" illustration $f(x)=i(x+i \varepsilon)^{2}$ returns us back to our "new-SUSY" example $V^{( \pm)}$which manifestly breaks the standard SUSY at $\varepsilon>0$.

\subsection{Intertwining relations}

Much in the same spirit as when one seeks possible interwinings of operators in the context of higher-order SUSY let us now return to their present realization, starting from the assumption of reality of the energies in the Schrödinger equation

$$
H^{(-)} \psi_{n}^{(-)}(x)=B^{(-)} A^{(-)} \psi_{n}^{(-)}(x)=E_{n}^{(-)} \psi_{n}^{(-)}(x)
$$

In the light of its possible re-factorization (8) let us now pre-multiply it by a suitable operator from the left,

$$
\left[\mathcal{T} A^{(-)}\right]\left[B^{(-)} \mathcal{T}\right]\left[\mathcal{T} A^{(-)} \psi_{n}^{(-)}(x)\right] \equiv H^{(+)} \psi_{m}^{(+)}(x)=E_{n}^{(-)}\left[\mathcal{T} A^{(-)} \psi_{n}^{(-)}(x)\right]
$$

This correspondence can be accompanied by the second Schrödinger equation

$$
H^{(+)} \psi_{m}^{(+)}(x)=B^{(+)} A^{(+)} \psi_{m}^{(+)}(x)=E_{m}^{(+)} \psi_{m}^{(+)}(x) .
$$

A comparison results in the general relationship

$$
\psi_{m}^{(+)}(x)=\mathcal{T} A^{(-)} \psi_{n}^{(-)}(x), \quad E_{m}^{(+)}=E_{n}^{(-)}
$$

In parallel, we can also re-write $H^{(-)} \psi_{k}^{(-)}(x)$ in the re-factorized form

$$
\left[B^{(-)} \mathcal{T}\right] \mathcal{T} A^{(-)}\left[B^{(-)} \mathcal{T} \psi_{m}^{(+)}(x)\right]=E_{m}^{(+)}\left[B^{(-)} \mathcal{T} \psi_{m}^{(+)}(x)\right] \equiv E_{k}^{(-)} \psi_{k}^{(-)}(x)
$$


and deduce that

$$
\psi_{k}^{(-)}(x)=B^{(-)} \mathcal{T} \psi_{m}^{(+)}(x), \quad E_{k}^{(-)}=E_{m}^{(+)} .
$$

One has to be careful with a quick assignment of the labels. In general, one cannot be sure about the ordering of the levels. In accord with several explicit examples [24] their various permutations could occur here in general. Fortunately, many rules concerning the ordering of levels in real potentials find their direct analogues in the complexified Sturm Liouville oscillation theorems [25]. Moreover, their "almost standard" form applies in the case of the present "asympotically almost real" examples (5) and (6). For them it is possible to show that $m=n=k$. In such a case the current rules of reconstruction of the partner spectra (the variety of constructive examples of which can be found in refs. [7]) can be restored in their full strength.

\section{Discussion}

We have seen that a core of applicability of our new form of the SUSY transformation to a complex force lies in our understanding of the normalizability of the wave functions in the $\mathcal{P} \mathcal{T}$ symmetric formalism. Still, even in this context many formal questions remain open. For example, due to a spontaneous breakdown of the $\mathcal{P} \mathcal{T}$

symmetry the energies can sometimes coalesce in the complex conjugated pairs [11]. The rigorous foundations of the reality of the spectra must be always scrutinized anew.

Mathematically, a key feature of the present construction lies in a difference in the arguments $x \pm i \varepsilon=r_{( \pm)}(x)$ of our model potentials, i.e., in the domains of definition of the Hamiltonians. This freedom admits a further generalization to all the integrations paths $r_{( \pm)}(x)$ which are coupled by the reflection with respect to the real axis or, in the present language, by the time reversal. In this sense the operator $\mathcal{T}$ plays a double role: To its original meaning of a reflection of the complex plane 
(for coordinates) one has to add its use in our innovated hypercharges and in the alternative factorization of $H^{(+)}$.

The relevance of the present proposal is enhanced by several unexpected observations. Firstly we notice that our supersymmetric mapping does not seem to require the current complementary comment about the missing partner of the zero energy bound state itself. This means that the Witten index [5] vanishes, $n_{B}-n_{F}=0$. Still, due to the broken hermiticity of our Hamiltonians, both zero-energy states remain normalizable so that the supersymmetry itself remains unbroken.

We may conclude that the present $\mathcal{P} \mathcal{T}$ symmetric formalism is quite different from its current Hermitian predecessors. It resembles the models with periodic potentials [26] and the higher order SUSY quantum mechanics in the irreducible case. Let us recall that in the latter context one also cannot express the intermediate Hamiltonian as a Hermitian operator [21].

Undoubtedly, the immediate relationship to the vanishing Witten index makes our construction very appealing. In a summary, we could now distinguish between the three different forms of SUSY. Firstly, one defines the standard one in a formalism using the real potentials and superpotentials [4]. Secondly, a use of the complex superpotentials and charges which are not Hermitian conjugate of each other forms

simply an opposite extreme [27]. Thirdly, our present formalism stays somewhere in between. It constrains the latter unrestricted freedom by the fairly nontrivial $\mathcal{P} \mathcal{T}$ symmetry but, in contrast to the current Witten's SUSY it is not restricted to non-negative Hamiltonian operators.

\section{Acknowledgements}

M. Z. acknowledges the hospitality of INFN and the University of Bologna and also the grant Nr. A 1048004 of GA AS CR. B. B. thanks Prof. C. Quesne for several fruitful discussions. 


\section{References}

[1] Y. A. Gelfand and E P Likhtman, JETP Lett. 13 (1971) 323;

P. Ramond, Phys. Rev. D 3 (1971) 2415;

A. Neveu and J. Schwarz, Nucl. Phys. B 31 (1971) 86.

[2] Supersymmetry in Physics, V. A. Kostelecky and D K Campbell, editors, North Holland, Amsterdam, 1985;

G. G. Blado, Theoretica Chimica Acta 94 (1998) 53;

G. Moore, N. Nekrasov and S. Shatashvili, Communications in Mathematical Physics 209 (2000) 77.

[3] H. P. Nilles, Phys. Rep. 110 (1984) 1;

H. E. Haber and G. L. Kane, Phys. Rep. 117 (1985) 75

[4] E. Witten, Nucl. Phys. B 188, 513 (1981)

[5] E. Witten, Nucl. Phys. B 202, 253 (1982).

[6] Y. Shadmi and Y. Shirman, Rev. Mod. Phys. 72 (2000) 25, with further references.

[7] F. Cooper, A. Khare and U. Sukhatme, Phys. Rep. 251, 267 (1995).

[8] A. Das and S. A. Pernice, Nucl. Phys. B 561 (1999) 357;

E. E. Donets, A. Pashnev, J. Juan Rosales and M. M. Tsulaia, Phys. Rev. D 61 (2000) 043512 .

[9] V. Buslaev and V. Grecchi, J. Phys. A: Math. Gen. 26, 5541 (1993)

[10] C. M. Bender and A. V. Turbiner, Phys. Lett. A 173, 442 (1993);

F. Cannata, G. Junker and J. Trost, Phys. Lett. A 246, 219 (1998). 
[11] C. M. Bender and S. Boettcher, Phys. Rev. Lett. 24, 5243 (1998).

[12] C. M. Bender, S. Boettcher and P. N. Meisinger, J. Math. Phys. 40, 2201 (1999).

[13] E. Caliceti, S. Graffi and M. Maioli, Commun. Math. Phys. 75, 51 (1980);

E. Delabaere and F. Pham, Phys. Lett. A 250, 25 and 29 (1998);

M. Znojil, Phys. Lett. A. 259 (1999) 220.

[14] J. J. Loeffel and A. Martin, CERN report no. CERN-TH 1167 (1971), unpublished;

J. J. Loeffel, A. Martin, B. Simon and A. Wightman, Phys. Lett. B 30, 656 (1969).

[15] C. M. Bender and T. T. S. Wu, Phys. Rev. 184, 1231 (1969);

F. Fernández, R. Guardiola, J. Ros and M. Znojil, J. Phys. A: Math. Gen. 31 (1998) 10105.

[16] Y. Sibuya, Global Theory of a Second Order Linear Ordinary Differential Equation with a Polynomial Coefficient (Noth Holland, Amsterdam, 1975);

C. M. Bender and K. A. Milton, Phys. Rev. D 55, R3255 (1997)

[17] V. I Kukulin, V. M. Krasnopol'sky and J. Horáček, Theory of resonances: Principles and Applications (Kluwer, Dordrecht, 1989);

G. Alvarez, J. Phys. A: Math. Gen. 27 (1995) 4589.

[18] C. M. Bender and S. Boettcher, J. Phys. A: Math. Gen. 31, L273 (1998).

[19] M. Znojil, J. Phys. A: Math. Gen. 27, 4945 (1994) and M. Znojil, arXiv: math-ph/0002036 (unpublished).

[20] A. G. Ushveridze, Quasi-exactly solvable models in quantum mechanics (IOPP, Bristol, 1994). 
[21] A. A. Andrianov, M. V. Ioffe and V. P. Spiridonov, Phys. Lett. A 174, 273 (1993);

A. A. Andrianov, M. V. Ioffe, F. Cannata and J. P. Dedonder, Int. J. Mod. Phys. A 10, 2683 (1995);

A. A. Andrianov, M. V. Ioffe and D. N. Nishnianidze, Theor. Math. Phys. 104, 1129 (1995);

A. A. Andrianov, M. V. Ioffe and F. Cannata, Mod. Phys. Lett. A 11, 1417 (1996).

[22] B. Bagchi and R. Roychoudhury, J. Phys. A: Math. Gen. 33 (2000) L1;

M. Znojil, J. Phys. A: Math. Gen. 33, L61 (2000).

[23] B. Bagchi, A. Ganguly, D. Bhaumik and A. Mitra, Mod. Phys. Lett. A14 (1999) 27.

[24] M. Znojil, Phys. Lett. A. 264 (1999) 108;

G. Lévai and M. Znojil, submitted and arXiv: quant-ph/0003081.

[25] E. Hille, Lectures on Ordinary Differential Equations, Addison-Wesley, Reading, 1969.

[26] G. V. Dunne and J. Feinberg, Phys. Rev. D 57, 1271 (1998).

[27] A. A. Andrianov, M. V. Ioffe, F. Cannata and J. P. Dedonder, Int. J. Mod. Phys. A 14, 2675 (1999). 